\title{
Intelligent Reflecting Surface Assisted Mobile Edge Computing for Internet of Things
}

\author{
Zheng Chu, Pei Xiao, Mohammad Shojafar, De Mi, Juquan Mao, and Wanming Hao
}

\begin{abstract}
This paper studies the impact of an intelligent reflecting surface (IRS) on computational performance in a mobile edge computing (MEC) system. Specifically, an access point (AP) equipped with an edge server provides MEC services to multiple internet of thing (IoT) devices that choose to offload a portion of their own computational tasks to the AP with the remaining portion being locally computed. We deploy an IRS to enhance the computational performance of the MEC system by intelligently adjusting the phase shift of each reflecting element. A joint design problem is formulated for the considered IRS assisted MEC system, aiming to optimize its sum computational bits and taking into account the CPU frequency, the offloading time allocation, transmit power of each device as well as the phase shifts of the IRS. To deal with the non-convexity of the formulated problem, we conduct our algorithm design by finding the optimized phase shifts first and then achieving the jointly optimal solution of the CPU frequency, the transmit power and the offloading time allocation by considering the Lagrange dual method and Karush-Kuhn-Tucker (KKT) conditions. Numerical evaluations highlight the advantage of the IRS-assisted MEC system in comparison with the benchmark schemes.
\end{abstract}

Index Terms-Intelligent reflecting surface (IRS), phase shifts, mobile edge computing (MEC), local computing, computational offloading.

\section{INTRODUCTION}

Internet of Things (IoT) technology realizes massive connectivity for the unprecedented proliferation of smart devices, and it is now developing towards intelligent sensing and communications for the deployment of smart environments, e.g., smart city, smart home, smart transportation, etc. [1]. In an IoT network, a massive number of wireless devices (WDs) (e.g., sensor nodes) are deployed to connect to an access point (AP) that is capable of sensing, communications and computations. These WDs are typically capacity-limited with energysaving low-performance processors, which have imposed a huge challenge to perform resource-intensive tasks in a lowlatency manner [2]. As a result, the self-sustainable and highperformance computational capabilities are two performance metrics to deal with the challenge in IoT systems.

Smart radio environment offers the seamless wireless connectivity and the capability of potentially processing and transmitting data by recycling the existing radio waves instead of generating new ones [3], as it provides a self-sustainable feature for the WDs. Intelligent reflecting surface (IRS) is considered as a novel enabler of smart radio environments, by intelligently controlling the scattering, reflection, and refraction characteristics of the radio waves to mitigate the negative effects introduced by wireless propagation [4], [5]. The IRS

This work was supported by the U.K. Engineering and Physical Sciences Research Council under Grants EP/P008402/2 and EP/ R001588/1. The work of W. Hao was supported by Scientific and Technological Key Project of Henan Province under Grant 202102210119.

Z. Chu, P. Xiao, M. Shojafar, D. Mi, and J. Mao are with the Institute for Communication Systems, University of Surrey, Guildford GU2 7XH, UK. (Email: andrew.chuzheng7@gmail.com, p.xiao@ surrey.ac.uk, m.shojafar@surrey.ac.uk,d.mi@surrey.ac.uk,juquan.mao@surrey.ac.uk)

W. Hao is with the School of Information Engineering, Zhengzhou University, Zhengzhou 450001, China (e-mail: iewmhao@zzu.edu.cn) is composed of massive reconfigurable reflecting elements, and coordinated by a software-oriented IRS controller. These reflecting elements typically have small, low-cost, and lowenergy consumption features, and each of which is able to coordinate the propagation environment of the intended signal via intelligently varying its amplitude and phase. Thus, the IRS can be utilized to enhance both spectral and energy efficiencies of wireless networks without extra energy consumption [6][11].

Mobile edge computing (MEC), on the other hand, is one of the promising techniques to improve the computational capability for WDs, by offloading partial or all of their own computational tasks from WDs to the MEC server APs in the vicinity in a low-latency fashion [2]. Via MEC, small-size and low-power WDs can offload their computational tasks to APs, and these tasks can be downloaded and computed remotely by the integrated MEC servers [12]. If the computational tasks are successfully offloaded, the MEC technique can facilitate the real-time implementation of computation-intensive tasks through both local computing on devices and edge computing of the offloading tasks on the MEC server [13]-[15]. Although the computational offloading in MEC systems can effectively support resource-intensive WDs, it is in practice difficult to unleash the full power of the MEC IoT, especially when the computational tasks cannot be successfully offloaded from the devices to the AP due to the propagation impairments of a communication link between them. In the recent work of [16] and [17], the IRS application to the MEC system has been considered one of the feasible solutions that can benefit the MEC during the offloading stage. In [16], [17], computational latency and energy consumption are considered as the performance metrics to evaluate the IRS assisted MEC systems, which only exploit the IRS's benefits to mitigate the computational latency and improve energy efficiency in MEC systems. In addition, the sum computational bits is considered as a direct performance metric to evaluate the overall computing capability of a MEC system. Although the existing MEC systems exploited the sum computational bits or computational efficiency maximization [14], [15], these works have not yet investigated the benefits of the IRS for MEC systems. As such, there is a major research gap on the application of the IRS to improve the computational offloading. To the authors' best knowledge, there is a paucity of work that considered the computational bits maximization in the IRS assisted MEC systems. These motivate our work in this paper.

This paper investigates an IRS enabled MEC system in the context of IoT, where partial computational offloading is adopted. The contributions of this paper are summarized as follows:

1) To evaluate the IRS assisted MEC system, we are interested in maximizing sum computational bits to exploit the IRS benefits on the overall computing performance of the MEC system. It aims to optimally design the CPU frequency, the offloading time allocation, the transmit power of each device, as well as the phase shifts of the 
IRS.

2) The formulated problem is not jointly convex due to multiple coupled variables. To deal with this non-convex problem, we first prove that the IRS's optimal phase shifts are independent of other variables, which can be derived in closed-form. Also, our work reveals that the IRS brings a significant enhancement of computational bits with an increasing number of reflecting elements.

3) We further derive the optimal closed-form CPU frequency as well as transmit power allocation of each device, respectively, by taking into consideration the Lagrange dual method and Karush-Kuhn-Tucker (KKT) conditions. Additionally, the optimal offloading time allocation is achieved via the bisection method.

\section{SySTEM MODEL}

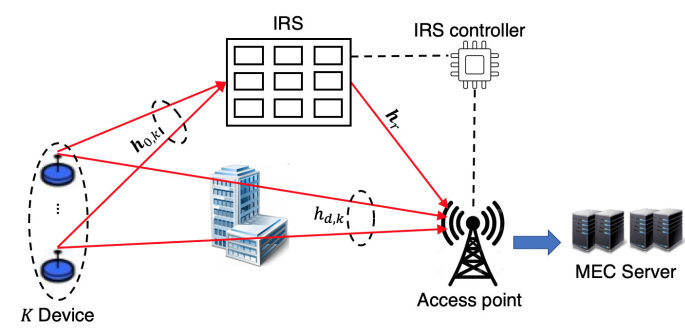

Fig. 1: An IRS assisted MEC system.

This section studies an IRS aided MEC system as shown in Fig. 1. To be specific, an AP, associated with an MEC server, provides edge computing services for $K$ devices. These devices offload a portion of their computational tasks for edge computing at the AP over wireless links. Due to high pathloss attenuation and blockage effects between the devices and AP, an IRS with $N$ reflecting elements is deployed to assist the computational offloading from the devices to the AP, via generating passive beamforming coordinated by the IRS controller. For the IRS reflection aided offloading, we denote $\boldsymbol{\Theta}_{k}=\operatorname{diag}\left[\beta_{k, 1} \exp \left(j \alpha_{k, 1}\right), \ldots, \beta_{k, N} \exp \left(j \alpha_{k, N}\right)\right], \forall k \in$ $[1, K], n \in[1, N]$ as the diagonal phase shift matrix during different time allocations, where $\alpha_{k, n} \in[0,2 \pi]$ and $\beta_{k, n} \in[0,1]$ are the phase shift and amplitude of the associated reflection coefficient, respectively. Each element of the IRS is typically designed to maximize the reflection strength and steer the reflection angle towards the MEC server. Thus, $\beta_{k, n}=1$ throughout this paper without loss of generality. In addition, the channel coefficients between the $k$-th device and the AP, the $k$-th device and the IRS, as well as the IRS and the AP are denoted by $h_{d, k} \in \mathbb{C}, \mathbf{h}_{0, k} \in \mathbb{C}^{1 \times N}, \mathbf{h}_{r} \in \mathbb{C}^{N \times 1}$, respectively. In this paper, the channel state information (CSI) is assumed to be perfectly known, this is due to the fact that we are interested in achieving an upper bound of the sum computational bits in the IRS assisted MEC system. The IRS controller coordinates its switching between two working modes, such as reception mode for channel estimation and reflection mode for data transmission, respectively [6], which also provides a real-time CSI feedback via this controller. Also, the robust beamforming was exploited to evaluate the system performance based on the imperfect cascaded CSI [18], [19].

\section{A. Partial Computational Offloading}

In this subsection, we consider the partial computational offloading, where a portion of computational tasks can be locally computed at the devices, while the remaining portion can be offloaded to the AP for edge computing [14], [15]. For the partial computation offloading, each device's computational tasks can be partitioned into local computing and computational offloading to the AP.

1) Local computation: We denote $f_{k}$ and $C$ as the CPU frequency of the $k$-th device and the number of cycles required for locally computing one bit of raw data at the CPU of each device, respectively. Also, let $r_{k}$ be the effective capacitance coefficient of the processor's chip at the $k$-th device, which relies on the chip architecture. According to [15], the computational bits locally executed at the $k$-th device is given by $R_{\mathrm{loc}, k}=\frac{T f_{k}}{C}$, and the energy consumption for the $k$-th device's local computing can be written as $E_{\mathrm{loc}, k}=\operatorname{Tr}_{c} f_{k}^{3}$.

2) Computational Offloading: In order to circumvent the interference among devices during the computational offloading, the time division multiple access (TDMA) protocol is adopted during the devices' computational offloading stage, where $K$ devices offload their own computational task to the AP one by one during each time slot. ${ }^{1}$. The total offloading time block is set to $T$, and each device offloads its own data during its allocated time slot $\tau_{k} \in[0, T]$, and $\sum_{k=1}^{K} \tau_{k} \leq T$. The computational offloading tasks of the $k$-th device includes raw data and communication overhead, i.e., encryption and packet header [13], [15]. Let $\nu_{k} R_{\text {off }, k}$ denote the number of the offloaded bits from the $k$-th device to the AP, where $R_{\text {off }, k}$ is the number of the raw data of the $k$-th device to be computed at the AP and $\nu_{k}$ is the communication overhead. In addition, $B$ is the bandwidth of the wireless link, $p_{k}$ is the transmit power of the $k$-th device, and $\sigma^{2}$ denotes the noise power. Thus, the maximum number of bits that the $k$ th device offloads to the MEC server at the AP is given by $R_{\text {off }, k}=\frac{\tau_{k} B}{\nu_{k}} \log _{2}\left(1+\frac{p_{k}\left|h_{d, k}+\mathbf{h}_{0, k} \boldsymbol{\Theta}_{k} \mathbf{h}_{r}\right|^{2}}{\sigma^{2}}\right)$. Also, the energy consumed for the $k$-th device's offloading is calculated as $E_{\text {off }, k}=\tau_{k}\left(p_{k}+p_{c, k}\right)$, where $p_{c, k}$ is a constant circuit power of the $k$-th device during its computational offloading.

\section{B. Problem Formulation}

In this subsection, we aim to maximize sum computational bits by jointly designing the CPU frequency, the offloading time allocations, the transmit power of each device, as well as the phase shifts of the IRS. To proceed, the formulated optimization problem is given by

$$
\begin{aligned}
\max _{\mathbf{p}, \boldsymbol{\tau}, \boldsymbol{\Theta}, \boldsymbol{f}} & \sum_{k=1}^{K} R_{\mathrm{off}, k}+\sum_{k=1}^{K} R_{\mathrm{loc}, k} \\
\text { s.t. } & \tau_{k}\left(p_{k}+p_{c, k}\right)+T r_{c} f_{k}^{3} \leq E_{\max }, \\
& \sum_{k=1}^{K} \tau_{k} \leq T, \quad 0 \leq \tau_{k} \leq T, \forall k \in[1, K], \\
& p_{k} \geq 0, f_{k} \geq 0, \forall k \in[1, K], \\
& \boldsymbol{\Theta}_{k}=\operatorname{diag}\left[\exp \left(j \alpha_{k, 1}\right), \ldots, \exp \left(j \alpha_{k, N}\right)\right],
\end{aligned}
$$

$\left|\exp \left(j \alpha_{k, n}\right)\right|=1, \alpha_{k, n} \in[0,2 \pi], \forall k \in[1, K], \forall n \in[1, N]$. (1d) where $\mathbf{p}=\left[p_{1}, \ldots, p_{K}\right], \boldsymbol{\tau}=\left[\tau_{1}, \ldots, \tau_{K}\right], \boldsymbol{\Theta}=\left[\boldsymbol{\Theta}_{1}, \ldots, \boldsymbol{\Theta}_{K}\right]$, $\boldsymbol{f}=\left[f_{1}, \ldots, f_{K}\right]$, and $E_{\max }$ denotes the maximum available energy consumption of each device.

\section{Optimal Solution to Problem (1)}

Problem (1) is not jointly convex due to coupling variables with respect to the CPU frequency, the offloading time allo-

\footnotetext{
${ }^{1}$ The whole offloading process consists of three stages, i.e., the offloading stage, the computing stage, and the downloading stage. Note that the computation and downloading stages can be negligible [12], [15].
} 
cation, the transmit power as well as the phase shifts of the IRS, which cannot be solved directly. To make this problem tractable, we propose a two-step approach to solve (1), and to be specific, it is split into two decoupled sub-problems. We first solve (1) to obtain the optimal phase shifts of the IRS, and then derive the optimal CPU frequency, transmit power, as well as the offloading time allocation via the Lagrange duality method and KKT conditions. To proceed, we present the following lemma to optimally design the phase shifts of the IRS at the different offloading time slots.

Lemma 1: The following problem is independently solved to achieve the optimal solution of the phase shift matrix $\boldsymbol{\Theta}_{k}$ during the $k$-th offloading time slot $\tau_{k}$

$$
\begin{gathered}
\max _{\boldsymbol{\Theta}_{k}}\left|h_{d, k}+\mathbf{h}_{0, k} \boldsymbol{\Theta}_{k} \mathbf{h}_{r}\right|^{2}, \text { s.t. }\left|\exp \left(j \alpha_{k, n}\right)\right|=1, \\
\alpha_{k, n} \in[0,2 \pi], \forall k \in[1, K], \forall n \in[1, N] .
\end{gathered}
$$

Proof: See Appendix A

To solve (2), we modify its objective function as

$$
\left|h_{d, k}+\mathbf{h}_{0, k} \boldsymbol{\Theta}_{k} \mathbf{h}_{r}\right|=\left|h_{d, k}+\boldsymbol{\theta}_{k} \mathbf{b}_{k}\right| \text {, }
$$

where $\mathbf{b}_{k}=\operatorname{diag}\left(\mathbf{h}_{0, k}\right) \mathbf{h}_{r}, \boldsymbol{\theta}_{k}=\left[\theta_{k, 1}, \ldots, \theta_{k, N}\right]=$ $\left[\exp \left(j \alpha_{k, 1}\right), \ldots, \exp \left(j \alpha_{k, N}\right)\right],\left|\theta_{k, n}\right|=1$. Next, by applying the triangle inequality, the upper bound of (3) can be derived as $\left|h_{d, k}+\boldsymbol{\theta}_{k} \mathbf{b}_{k}\right| \leq \sum_{n=1}^{N}\left|h_{d, k}\right|+\left|\theta_{k, n} \mathbf{b}_{k}[n]\right|=\sum_{n=1}^{N}\left|h_{d, k}\right|+$ $\left|\mathbf{b}_{k}[n]\right|$, where $\mathbf{b}_{k}[n]$ is the $n$-th element of $\mathbf{b}_{k}$, and equality holds with $\left|\theta_{k, n}\right|=1$ for $n \in[1, N]$. We obtain the upper bound via

$$
\alpha_{k, n}=\arg \left(h_{d, k}\right)-\arg \left(\mathbf{b}_{k}[n]\right),
$$

where $\arg (*)$ denotes the phase operator. Thus, the optimal phase shift vector can be obtained from (4), denoted by $\boldsymbol{\theta}_{k}^{*}$, and equivalently the optimal phase shift matrix $\Theta_{k}^{*}$ is recovered from $\boldsymbol{\theta}_{k}^{*}$. For the optimal phase shift matrix $\Theta_{k}^{*}$, the following proposition is required.

Proposition 1: The optimal phase shift matrix $\Theta_{k}^{*}$ aligns the cascaded link between the $k$-th device and the AP via the IRS with the direct link, which can be expressed as $\mathbf{h}_{0, k} \boldsymbol{\Theta}_{k}^{*} \mathbf{h}_{r}=$ $\xi_{k} h_{d, k}, \forall k \in[1, K]$, where $\xi_{k}$ is positive scalar.

Proof: See Appendix B

By exploiting Proposition 1 , the following remark is required to quantify the signal strength of information reflection during the offloading stage.

Remark 1: The IRS is deployed to strengthen the received signal power of the $k$-th device during its offloading stage at most $\left(1+\xi_{k}\right)^{2}$ times in comparison to that without IRS. In addition, the signal strength of the $k$-th device $\xi_{k}$ is proportional to the number of reflecting elements of the IRS. Hence, a significant improvement can be introduced during the offloading stage with an increased number of reflecting elements.

After optimally designing the phase shifts of the IRS, we consider jointly optimization of the CPU frequency, the offloading time allocation, as well as the power allocation. Let $t_{k}=\frac{\left|h_{d, k}+\mathbf{h}_{0, k} \mathbf{\Theta}_{k}^{*} \mathbf{h}_{r}\right|^{2}}{\sigma^{2}}$, and $\tilde{p}_{k}=\tau_{k} p_{k}$, problem (1) can be rewritten as

$$
\begin{aligned}
\max _{\tilde{\mathbf{p}}, \boldsymbol{\tau}, \boldsymbol{f}} & \sum_{k=1}^{K} \frac{\tau_{k} B}{\nu_{k}} \log _{2}\left(1+\frac{\tilde{p}_{k} t_{k}}{\tau_{k}}\right)+\sum_{k=1}^{K} \frac{T f_{k}}{C}, \\
\text { s.t. } & \tilde{p}_{k}+\tau_{k} p_{c, k}+T r_{c} f_{k}^{3} \leq E_{\max }, \\
& \sum_{k=1}^{K} \tau_{k} \leq T, \quad 0 \leq \tau_{k} \leq T, \forall k \in[1, K], \\
& \tilde{p}_{k} \geq 0, f_{k} \geq 0, \forall k \in[1, K],
\end{aligned}
$$

where $\tilde{\boldsymbol{p}}=\left[\tilde{p}_{1}, \ldots, \tilde{p}_{K}\right]$. Problem (5) can be proved to be jointly convex in terms of the CPU frequency, the transmit power, as well as the offloading time allocation [20]. Thus, it can be solved by the interior-point method [21]. To gain more engineering insights, the Lagrange dual method is employed to derive its optimal solution in terms of closed-form expression. To proceed, the Lagrange dual function of problem (5) is written as

$$
\begin{aligned}
& \mathcal{L}\left(\lambda_{k}, \mu, \tilde{\mathbf{p}}, \boldsymbol{\tau}, \boldsymbol{f}\right)=\sum_{k=1}^{K}\left[\frac{\tau_{k} B}{\nu_{k}} \log _{2}\left(1+\frac{\tilde{p}_{k} t_{k}}{\tau_{k}}\right)+\frac{T f_{k}}{C}\right] \\
& -\sum_{k=1}^{K} \lambda_{k}\left(\tilde{p}_{k}+\tau_{k} p_{c, k}+T r_{c} f_{k}^{3}-E_{\max }\right)-\mu\left(\sum_{k=1}^{K} \tau_{k}-T\right),
\end{aligned}
$$

where $\boldsymbol{\lambda}=\left[\lambda_{1}, \ldots, \lambda_{K}\right]$ and $\mu$ denote the dual multipliers of constraints (5b) and (5c), respectively. Also, the dual problem of (5) is given by

$$
\min _{(\tilde{\mathbf{p}}, \boldsymbol{\tau}, \boldsymbol{f}) \in \mathcal{S}} \mathcal{L}\left(\lambda_{k}, \mu, \tilde{\mathbf{p}}, \boldsymbol{\tau}, \boldsymbol{f}\right) .
$$

Note that $\mathcal{S}$ is the feasible set of $(\tilde{\mathbf{p}}, \boldsymbol{\tau}, \boldsymbol{f})$, which has been shown in constraints (5b) and (5c). Problem (5) is convex and satisfies Slaters condition, and the optimal solution of (5) satisfies the KKT conditions [21]. To derive the optimal $\mathrm{CPU}$ frequency and transmit power, the following theorem is proposed.

Theorem 1: The optimal CPU frequency $f_{k}$ and transmit power $p_{k}$ are derived in terms of closed-form solution, respectively, as

$$
\begin{aligned}
& f_{k}^{*}=\left(\frac{T}{3 \lambda_{k} r_{c} C}\right)^{\frac{1}{2}}, \\
& p_{k}^{*}=\left\{\begin{array}{cc}
0 & \tau_{k}=0 \\
{\left[\frac{B}{\lambda_{k} \ln 2}-\frac{1}{t_{k}}\right]^{+}} & \text {otherwise }
\end{array}\right.
\end{aligned}
$$

Proof: See Appendix C

Next, we design the offloading time allocation, where the firstorder derivative of (6) is derived with respect to $\tau_{k}$ and set it to zero, i.e., $\frac{\partial \mathcal{L}}{\partial \tau_{k}}=0$. From [14], we solve the following equation via employing bisection method [21] to obtain the optimal offloading time at the $k$-th device, i.e.,

$$
\log _{2}\left(1+\frac{\tilde{p}_{k} t_{k}}{\tau_{k}}\right)-\frac{\tilde{p}_{k} t_{k}}{\left(\tau_{k}+\tilde{p}_{k} t_{k}\right) \ln 2}=\frac{\lambda_{k} p_{c, k}+\mu}{B} .
$$

Moreover, the sub-gradient method is adopted to iteratively update the dual variables. The sub-gradient method is written as

$$
\begin{aligned}
& \left(\lambda_{k}(m+1), \mu(m+1)\right)=\left(\lambda_{k}(m), \mu(m)\right) \\
& -\left(\phi_{1}(m), \phi_{2}(m)\right) \cdot\left(\Delta \lambda_{k}(m), \Delta \mu(m)\right),
\end{aligned}
$$

where $m$ is the iteration index, $\phi_{1}(m)$ and $\phi_{2}(m)$ represent the $m$-th iterative step. Also, $\Delta \lambda_{k}(m)$ and $\Delta \mu(m)$ are expressed, respectively, as $\Delta \lambda_{k}(m)=E_{\max }-$ $\left[\tilde{p}_{k}^{*}(m)+\tau_{k} p_{c, k}+\operatorname{Tr}_{c}\left(f_{k}^{*}(m)\right)^{3}\right]$, and $\Delta \mu(m)=T-$ $\sum_{k=1}^{K} \tau_{k}^{*}(m)$, where $f_{k}^{*}(m), \tilde{p}_{k}^{*}(m)$, and $\tau_{k}^{*}(m)$ denote the optimal solutions at the $m$-th iteration. The proposed two-step approach to solve problem (1) is summarized in Algorithm 1. By exploiting Algorithm [1, the optimal CPU frequency, transmit power, and the offloading time allocation can be achieved. According to [21], it is verified that the sub-gradient method guarantees its solution convergence.

Algorithm 1: The proposed algorithm to solve problem (1)

1) Initialization: The iterative number $m=1, \lambda_{k}(m) \geq 0$, $\mu(m) \geq 0$.

2) Obtain the optimal phase shift matrix $\boldsymbol{\Theta}_{k}$ via solving problem (2).

3) Repeat 
a) Obtain the optimal CPU frequency $f_{k}^{*}(m)$ and $p_{k}^{*}(m)$ via Theorem 1 for given $\lambda_{k}(m)$ and $\mu(m)$.

b) Calculate the optimal offloading time allocation $\tau_{k}(m)$ via bisection method to solve equation (9).

c) Update dual variables $\lambda_{k}(m)$ and $\mu(m)$ via the sub-gradient method (10).

d) Update iteration index $m=m+1$.

4) Until convergence

\section{NumericAl RESULts}

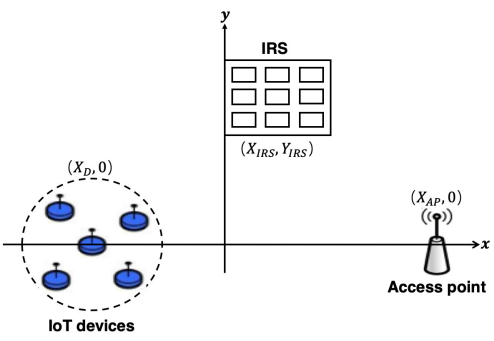

Fig. 2: System deployment.

In this section, numerical evaluations are demonstrated to highlight the benefits of the proposed IRS-assisted MEC system. We consider a two-dimensional (2-D) coordinates to describe the network topology as shown in Fig. 2. where the IRS and the AP are located as $\left(X_{\mathrm{IRS}}, Y_{\mathrm{IRS}}\right)$ and $\left(X_{\mathrm{AP}}, 0\right)$, the users are randomly located within a circular area centered at $\left(X_{\mathrm{D}}, 0\right)$ with radius $2 \mathrm{~m}$. Specifically, $Y_{\mathrm{IRS}}=2 \mathrm{~m}$, $X_{\mathrm{AP}}=20 \mathrm{~m}$, and $X_{\mathrm{D}}=-10 \mathrm{~m}$. The channel coefficient consists of distance-dependent path loss model and small-scale fading, where the path loss model is set to $P_{L}=A d^{-\kappa}$, where $A=-30 \mathrm{~dB}$ is the path-loss at the reference distance $1 \mathrm{~m}, \kappa$ is the path loss exponent, and $d$ represents the distance between any two devices, i.e., the devices and the IRS, the devices and the AP, as well as the IRS and the AP. The path loss exponents of devices-AP, devices-IRS, and IRS-AP are set to $3.5,2.5$, and 2.5 , respectively. Also, the reflecting elements of the IRS $N=50$, the number of device $K=5$, the system bandwidth $B=2 \mathrm{MHz}$, the capacitance coefficient $r_{c}=10^{-26}$, the noise power $\sigma^{2}=10^{-9} \mathrm{~W}$, the number of cycles for one bit $C=10^{3}$ cycle/bit, the maximum available energy consumption of each device $E_{\max }=15 \mathrm{dBm}$, and the circuit power $p_{c, k}=p_{c}=10^{-4} \mathrm{~W}$. We compare the proposed scheme with the existing benchmark schemes under the same configurations [15]: partial offloading scheme without IRS; offloading scheme only with/without IRS; local computing only.

First, we evaluate the impact of the maximum available energy consumption during computational offloading of the devices on the performance of the IRS-assisted MEC system. Fig. 3(a) shows that the sum computational bits versus maximum available energy consumption of each device $E_{\max }$. From this figure, it is shown that the number of computational bits increases with $E_{\max }$. Also, the proposed partial offloading scheme outperforms the other benchmark schemes, and the gap between them becomes larger as $E_{\max }$ increases. This is due to the fact that the devices can intelligently allocate resources for computational offloading and local computing. Meanwhile, thanks to the IRS, more energy consumption strengthens the signal reception and reflection efficiency such that the proposed schemes can effectively improve the computational offloading bits in comparison to its counterpart without IRS.
In Fig. 3(b), the performance of sum computational bits is illustrated with the different circuit power of the devices $p_{c}$, where the sum computational bits has a moderately decreasing trend as $p_{c}$ increases. This figure reveals the fact that an increased circuit power of the devices yields an adverse impact on the computational bits and degrades the computational offloading performance of the IRS assisted MEC system. Also, the sum computational bits are more sensitive in the larger circuit power region. Additionally, the proposed partial offloading scheme exhibits its robustness in comparison to the other benchmark schemes. This may be explained by the fact that the use of the IRS in computational offloading outweighs the performance loss incurred by increasing circuit power of the device, which highlights the importance of the IRS in the MEC system.

Then, we present the sum computational bits versus the reflecting elements of the IRS $N$ in Fig. 3(c). From this figure, it is observed that the IRS plays a significant role in improving the computational bits in comparison to its counterparts without IRS. This is due to the fact that the computational offloading can be effectively enhanced via the IRS-assisted wireless transmission, especially with larger reflecting elements, which validates the theoretical discussion in Remark 11. In Fig. 3(d), the sum computational bits versus the $\mathrm{x}$-coordinate of the IRS $X_{\text {IRS }}$ is demonstrated to evaluate the impact of the IRS deployment on the performance of the IRS-assisted MEC system. This result suggests that the IRS should be deployed in the close proximity to the device or the AP, which effectively enhance the sum computation bits. Moreover, the IRS assisted schemes outperforms its counterparts without IRS, which are not affected by the IRS deployment, in terms of computational bits.

\section{CONClusion}

This paper investigated an IRS assisted MEC system. To evaluate its computational performance, we considered maximizing the sum computational bits to optimally design the CPU frequency, the transmit power and time allocation of computational offloading, as well as the phase shifts of the IRS. We first derived the phase shifts of the IRS in a closed-form expression which is independent of other variables, and proved that a significant improvement could be achieved by using the IRS for the computational offloading stage. Then, the optimal CPU frequency and transmit power of each device were derived via the Lagrange dual method and KKT conditions, and the bisection method was adopted to solve the offloading time allocation optimally. Moreover, we employed the sub-gradient method to update the dual variables iteratively. The numerical results have demonstrated the benefits of the IRS in the MEC system, in comparison to the benchmark schemes. For future work, we could consider the IRS assisted wireless powered MEC to address energyconstrained issue of the devices and other multiple access schemes, i.e., non-orthogonal multiple access (NOMA) and frequency division multiple access (FDMA). Also, the quantized phase shifts would be more practical than the continuous ones. In addition, we could investigate minimizing latency and energy consumption of the IRS aided MEC system in our future work.

\section{APPENDIX}

\section{A. Proof of Lemma 1}

It is easily verified that the achievable throughput of the $k$-th device (i.e., $R_{\mathrm{off}, k}$ ) is an monotonically increasing 


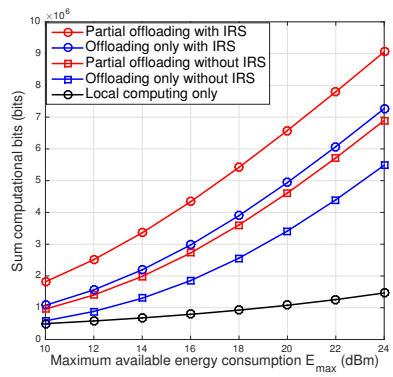

(a) Sum computational bits versus $E_{\max }$.

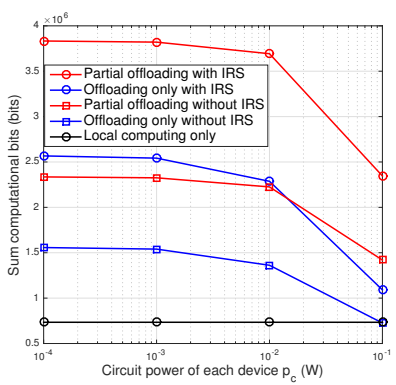

(b) Sum computational bits versus $p_{c}$.
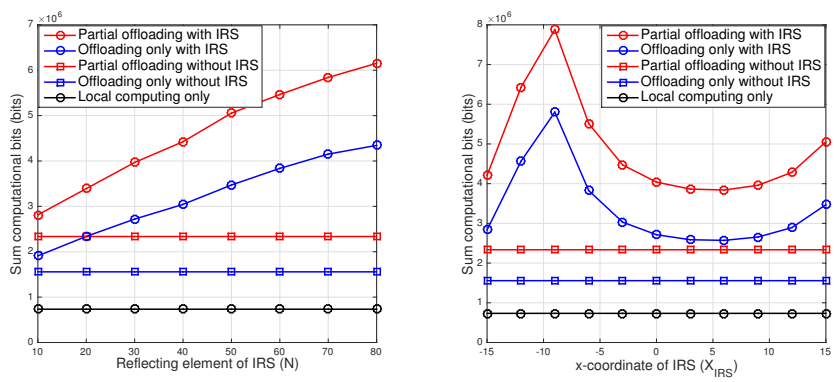

(c) Sum computational bits versus $N$.(d) Sum computational bits versus $X_{\text {IRS }}$.

Fig. 3: Numerical evaluations of sum computational bits.

function in $\left|h_{d, k}+\mathbf{h}_{0, k} \boldsymbol{\Theta}_{k} \mathbf{h}_{r}\right|^{2}$. Thus, maximizing problem (1) is equivalent to the maximization of $\left|h_{d, k}+\mathbf{h}_{0, k} \boldsymbol{\Theta}_{k} \mathbf{h}_{r}\right|^{2}$ for $\forall k \in[1, K]$, each of which only relies on $\boldsymbol{\Theta}_{k}$ with $\left|\exp \left(j \alpha_{k, n}\right)\right|=1$. Thus, we can independently solve problem (2) to obtain the optimal phase shift matrix $\boldsymbol{\Theta}_{k}$, which completes Lemma 1.

\section{B. Proof of Proposition 1}

With some mathematical manipulations, the term $\left|h_{d, k}+\mathbf{h}_{0, k} \boldsymbol{\Theta}_{k}^{*} \mathbf{h}_{r}\right|^{2}$ can be expressed as

$\left|h_{d, k}+\mathbf{h}_{0, k} \boldsymbol{\Theta}_{k}^{*} \mathbf{h}_{r}\right|^{2}=\left|h_{d, k}\right|^{2}+\left|\mathbf{h}_{0, k} \Theta_{k}^{*} \mathbf{h}_{r}\right|^{2}$

$+2\left|\mathbf{h}_{0, k} \boldsymbol{\Theta}_{k}^{*} \mathbf{h}_{r}\right|\left|h_{d, k}\right| \cos \left[\arg \left(h_{d, k}\right)-\arg \left(\mathbf{h}_{0, k} \Theta_{k}^{*} \mathbf{h}_{r}\right)\right]$. (11)

From (11), it is easily verified that $\left|h_{d, k}+\mathbf{h}_{0, k} \boldsymbol{\Theta}_{k}^{*} \mathbf{h}_{r}\right|^{2}$ achieves its maximum value if $\cos \left[\arg \left(h_{d, k}\right)-\arg \left(\mathbf{h}_{0, k} \boldsymbol{\Theta}_{k}^{*} \mathbf{h}_{r}\right)\right]=1$. It means that the phases of both direct and cascaded links between the $k$-th device and the AP are identical, i.e., $\arg \left(h_{d, k}\right)=\arg \left(\mathbf{h}_{0, k} \boldsymbol{\Theta}_{k}^{*} \mathbf{h}_{r}\right)$, which completes Proposition 1

\section{Proof of Theorem 1}

To derive $8 \mathrm{aa}$ and $8 \mathrm{a}$, we take into consideration the firstorder derivatives of 6 with respect to $f_{k}$ and $\tilde{p}_{k}$, respectively, and let them to zero, which are expressed as

$$
\begin{aligned}
& \frac{\partial \mathcal{L}}{\partial f_{k}}=\frac{T}{C}-3 \lambda_{k} r_{c} f_{k}^{2}=0, \\
& \frac{\partial \mathcal{L}}{\partial \tilde{p}_{k}}=\frac{B t_{k}}{\left(1+\frac{\tilde{p}_{k} t_{k}}{\tau_{k}}\right) \ln 2}-\lambda_{k}=0 .
\end{aligned}
$$

From $12 \mathrm{a}$, the optimal CPU frequency can be derived as $f_{k}^{*}=\left(\frac{T}{3 \lambda_{k} r_{c} C}\right)^{\frac{1}{2}}$. By $12 \mathrm{~b}$ and $\tilde{p}_{k}=\tau_{k} p_{k}$, we have the closed-form power allocation $p_{k}^{*}=\left[\frac{B}{\lambda_{k} \ln 2}-\frac{1}{t_{k}}\right]^{+}$.In addition, if the $k$-th device does not perform computational offloading, i.e., $\tau_{k}=0$, it correspondingly leads to no transmit power consumed (i.e., $p_{k}=0$ ). Thus, we completes proof of Theorem 1

\section{REFERENCES}

[1] F. Zhou, Y. Wu, R. Q. Hu, Y. Wang, and K. K. Wong, "Energyefficient NOMA enabled heterogeneous cloud radio access networks," IEEE Network, vol. 32, no. 2, pp. 152-160, Mar.-Apr. 2018.

[2] Y. Mao, C. You, J. Zhang, K. Huang, and K. B. Letaief, "A survey on mobile edge computing: The communication perspective," IEEE Commun. Surveys Tuts., vol. 19, no. 4, pp. 2322-2358, Fourth Quarter, 2017.

[3] Renzo, Marco Di, et. al., "Smart radio environments empowered by reconfigurable AI meta-surfaces: an idea whose time has come," EURASIP J. Wirel. Commun. Netw., no. 129, pp. 1-20, May 2019.
[4] Q. Wu and R. Zhang, "Towards smart and reconfigurable environment: Intelligent reflecting surface aided wireless network," IEEE Commun. Mag., vol. 58, no. 1, pp. 106-112, Jan. 2020.

[5] M. D. Renzo, A. Zappone, M. Debbah, M. Alouini, C. Yuen, J. D. Rosny, and S. Tretyakov, "Smart radio environments empowered by reconfigurable intelligent surfaces: How it works, state of research, and road ahead," IEEE J. Sel. Area Commun., pp. 1-1, 2020.

[6] Q. Wu and R. Zhang, "Intelligent reflecting surface enhanced wireless network via joint active and passive beamforming," IEEE Trans Wireless Commun., vol. 18 , no. 11, pp. 5394-5409, Nov. 2019.

[7] C. Huang, A. Zappone, G. C. Alexandropoulos, M. Debbah, and C. Yuen, "Reconfigurable intelligent surfaces for energy efficiency in wireless communication," IEEE Transactions on Wireless Communications, vol. 18, no. 8, pp. 4157-4170, Aug. 2019.

[8] C. Pan, H. Ren, K. Wang, W. Xu, M. Elkashlan, A. Nallanathan, and L. Hanzo, "Multicell MIMO communications relying on intelligent reflecting surfaces," IEEE Trans. Wireless Commun., vol. 19, no. 8, pp. 5218-5233, Aug. 2020

[9] G. Zhou, C. Pan, H. Ren, K. Wang, and A. Nallanathan, "Intelligent reflecting surface aided multigroup multicast miso communication systems," IEEE Trans. Signal Process., vol. 68, pp. 3236-3251, Apr. 2020.

[10] Z. Chu, W. Hao, P. Xiao, and J. Shi, "Intelligent reflecting surface aided multi-antenna secure transmission," IEEE Wireless Commun. Lett., vol. 9, pp. 108-112, Jan 2020.

[11] C. Pan, H. Ren, K. Wang, M. Elkashlan, A. Nallanathan, J. Wang, and L. Hanzo, "Intelligent reflecting surface aided MIMO broadcasting for simultaneous wireless information and power transfer," IEEE J. Sel. Areas Commun., vol. 38, no. 8, pp. 1719-1734, Aug. 2020.

[12] F. Wang, J. Xu, X. Wang, and S. Cui, "Joint offloading and computing optimization in wireless powered mobile-edge computing systems," IEEE Trans. Wireless Commun., vol. 17, no. 3, pp. 1784-1797, Mar. 2018.

[13] S. Bi and Y. J. Zhang, "Computation rate maximization for wireless powered mobile-edge computing with binary computation offloading," IEEE Trans. Wireless Commun., vol. 17, no. 6, pp. 4177-4190, Jun. 2018.

[14] F. Zhou, Y. Wu, R. Q. Hu, and Y. Qian, "Computation rate maximization in UAV-enabled wireless-powered mobile-edge computing systems," IEEE J. Sel. Area Comm., vol. 36, no. 9, pp. 1927-1941, Sept. 2018.

[15] F. Zhou and R. Q. Hu, "Computation efficiency maximization in wireless-powered mobile edge computing networks," IEEE Tran. Wireless Comm., vol. 19, no. 5, pp. 3170-3184, May 2020.

[16] T. Bai, C. Pan, Y. Deng, M. Elkashlan, A. Nallanathan, and L. Hanzo, "Latency minimization for intelligent reflecting surface aided mobile edge computing," to appear in IEEE J. Sel. Area Commun, pp. 1-1, 2020.

[17] T. Bai, C. Pan, H. Ren, Y. Deng, M. Elkashlan, and A. Nallanathan, "Resource allocation for intelligent reflecting surface aided wireless powered mobile edge computing in OFDM systems," https://arxiv.org/ $a b s / 2003.05511 .2020$.

[18] G. Zhou, C. Pan, H. Ren, K. Wang, M. D. Renzo, and A. Nallanathan, "Robust beamforming design for intelligent reflecting surface aided MISO communication systems," IEEE Wireless Commun. Lett., vol. 9, no. 10, pp. 1658-1662, Oct. 2020.

[19] G. Zhou, C. Pan, H. Ren, K. Wang, and A. Nallanathan, "A framework of robust transmission design for IRS-aided MISO communications with imperfect cascaded channels," IEEE Trans. Signal Process., vol. 68, pp. 5092-5106, Aug. 2020.

[20] Z. Chu, F. Zhou, Z. Zhu, R. Q. Hu, and P. Xiao, "Wireless powered sensor networks for internet of things: Maximum throughput and optimal power allocation," IEEE Internet Things J., vol. 5, no. 1, pp. 310-321, Feb. 2018

[21] S. Boyd and L. Vandenberghe, Convex Optimization. Cambridge, UK: Cambridge University Press, 2004. 